\title{
The Rotterdam study: why fall in COPD?
}

\author{
To the Editor:
}

LAHOUSSE et al. [1] have conducted an interesting study to investigate the association of chronic obstructive pulmonary disease (COPD) with various gait domains and explored a potential risk with falling. They have stressed the temporal gait parameter (rhythm) and have concluded that people who fall with COPD had worse rhythm. However, they have not tried to evaluate the other probable causes of disturbed rhythm in their subjects. The causes resulting in falls or disordered rhythm in COPD patients, could also be present primarily in the musculoskeletal and neurological systems because most of the subjects enrolled in the study were elderly, and problems related to musculoskeletal and neurological systems are more common in an elderly population [2]. The exclusion of such groups of people from the study has not been mentioned in the exclusion criteria. There were 152 participants with physical inability but the nature of physical inability is obscure. It would have been more rational or rather elucidative to assess other probable causes resulting in falls or decreased cadence in such elderly population with COPD.

Human gait is a complex interaction of hip, knee, ankle and foot motion to advance the body in the desired line of progression [3]. It can be assessed qualitatively (visual analysis) or quantitatively whereby the kinetics (forces producing motion), poly-electromyogram or dynamic electromyogram (analysis of muscle activity), kinematics (motion analysis) and energetics (analysis of metabolic or mechanical energy) can be assessed [4]. The purpose of gait analysis is, after all, the proper understanding, diagnosis and treatment of the primary cause of the overall gait problem. The possible causes that would have led to the disturbed rhythm could be: abnormal base of support (resulting from equinovarus foot, equinovalgus foot, flexion deformity of toes and hitchhikers' toe), Joint instability (ankle instability caused by insufficient plantar-flexion knee extension coupling, knee instability in the form of flexion or hyperextension, hip instability in the form of excessive hip flexion or adduction and trunk instability), problems in limb clearance (stiff knee, excessive pelvic obliquity or a drop foot) and musculosleletal pain [3,5-8].

The mean age of the participants in the study was $>70$ years and 100 of them had moderate-to-severe COPD with episodes of exacerbation. It is well known that such COPD patients are more likely to develop hypoxaemia and hypercapnia [9]. COPD patients with hypercapnia may present with drowsiness, mental cloudiness and tremours and this in turn may affect the gait of such persons. However, none of the participants was assessed for hypercapnia in the study. Besides, signal abnormality in cerebral white matter and impaired cerebral blood flow regulation are other strong risk factors for slow gait and falls in the elderly [10].

Therefore, the association of worse rhythm and pace including low cadence and gait velocity in the Rotterdam study could have been linked to even more factors which have not been enumerated. All such probable causes could have been assessed by kinetic, kinematic and dynamic electromyogram recording. Such evaluation could have explored a broader explanation of falls, especially in the elderly population. Thus, further extensive studies are required to conclude an association between COPD patients with decreased rhythm of gait.

0 @ERSpublications

Association of poor gait and falling in Rotterdam study may be due to factors that were not considered http://ow.ly/RZbUI

Pampa De ${ }^{1}$ and Sourin Bhuniya ${ }^{2}$

${ }^{1}$ Dept of Physical Medicine and Rehabilitation, College of Medicine and Sagar Dutta Hospital, Kolkata, India.

${ }^{2}$ Pulmonary Medicine, All India Institute of Medical Sciences (AIIMS), Bhubaneswar, India.

Correspondence: Sourin Bhuniya, Dept of Pulmonary Medicine, All India Institute of Medical Sciences (AIIMS) Bhubaneswar, Sijua, Dumduma, Bhubaneswar, 751019 India. E-mail: sbhuniya@hotmail.com

Received: July 252015 | Accepted after revision: July 312015

Conflict of interest: None declared.

\section{References}

1 Lahousse L, Verlinden VJ, van der Geest JN, et al. Gait patterns in COPD: the Rotterdam study. Eur Respir J 2015; 46: 88-95.

2 Sinaki M. Musculoskeletal challenges of osteoporosis. Aging (Milano) 1998; 10: 249-262. 
3 Perry J. Normal and pathological gait. In: Hsu JD, Michael JW, Fisk JR, eds. AAOS Atlas of Orthoses and Assistive Devices. Philadelphia, Mosby Elsevier, 2008; pp. 61-82.

4 Esquenazi A, Talaty M. Gait analysis: technology and clinical applications. In: Braddon RL, ed. Physical Medicine and Rehabilitation 3rd Edn. Saunders Elsevier, 2008; pp. 99-116.

5 Saunders JB, Inman VT, Eberhart HD. The major determinants in normal and pathological gait. J Bone Joint Surg Am 1953; 35-A: 543-558.

6 Mayer N, Keenan M, Esquenazi A. Limbs with restricted or excessive motion after traumatic brain injury. In: Rosenthal M, Griffith ER, Kreutzer GS, eds. Rehabilitation of the adult and child with traumatic brain injury. 3rd Edn. Philadelphia, Fa Davis, 1990; pp. 503-535.

7 Tom FN. Orthoses for cerebral palsy. In: Hsu JD, Michael JW, Fisk JR, eds. AAOS Atlas of Orthoses and Assistive Devices. Philadelphia, Mosby Elsevier, 2008; pp. 487-497.

8 Eyring EJ, Murray WR. The effect of joint position on the pressure of intra articular effusion. J Bone Joint Surg Am 1964; 46: 1235-1241.

9 Calverley PM. Respiratory failure in chronic obstructive pulmonary disease. Eur Respir J 2003; 22: Suppl. 47, $26 \mathrm{~s}-30 \mathrm{~s}$.

10 Sorond FA, Galica A, Serrador JM, et al. Cerebrovascular hemodynamics, gait and falls in an elderly population. Neurology 2010; 74: 1627-1633.

From the authors:

We thank De and Bhuniya for their interesting comments on our study that investigated gait patterns in chronic obstructive pulmonary disease (COPD) [1]. We measured 30 gait parameters during normal walking, turning and tandem walking in 1500 persons (mean age 74 years), and summarised these parameters into seven independent gait domains. Importantly, only the gait domain Rhythm (reflecting how quickly steps are taken) was significantly impaired in persons with COPD $[1,2]$. We fully support the call to investigate the causes of this disturbed Rhythm in COPD thoroughly and to elucidate why fallers with COPD exhibit especially worse Rhythm.

De and Bhuniya suggest four interesting probable causes for the disturbed Rhythm that we observed in persons with COPD: an abnormal base of support, joint instability, problems in limb clearance and musculoskeletal pain. However, abnormal base of support, joint instability or problems in limb clearance are more likely to affect gait domains such as Base of Support, Variability or Phases (reflecting double support time). Although we did hypothesise that both spatiotemporal and balance aspects of gait would be worse in COPD subjects compared with persons with normal lung function, we only observed disturbances in the temporal aspect of gait, Rhythm. This sole finding of an association for COPD with Rhythm reduces the probability that base of support, joint instability and problems in limb clearance are the cause or an intermediate of the associations found. The fourth suggested cause, musculoskeletal pain, may be more likely, since we observed that among participants of the Rotterdam Study, leg pain was found to associate significantly with worse Rhythm, independent from osteoarthritis [3].

Gait variability is a well-established risk factor for falls in elderly subjects and patients with neurodegenerative diseases [4]. In persons with COPD, lower limb muscle weakness and impaired activities of daily living predict falls, though little was known on the influence of gait deficits [5]. Our study added to the field that fallers with COPD exhibit worse Rhythm, while Rhythm did not discriminate fallers in persons without COPD. In addition, adjusting for cognitive status hardly changed associations between COPD and gait. These observations rather suggest that gait deficits in patients with COPD are different from natural ageing or common neurodegenerative diseases.

Finally, hypercapnia is not expected to be the principal cause of the slower Rhythm in our study as the COPD subjects mainly had mild to moderate disease (mean forced expiratory volume in $1 \mathrm{~s} 81 \%$ predicted, diffusing capacity of the lung for carbon monoxide $89 \%$ and $12 \%$ frequent exacerbators), generally visited our research centre in a stable phase and were not persuaded to perform the test if they felt physically too poor to walk (explaining the overall exclusion of 152 participants due to physical inability). Nevertheless, since COPD severity correlated with poorer Rhythm and more gait deficits (including smaller steps), we fully support the call for more pathophysiological studies to elucidate the role of hypoxaemia, hypercapnia and potential underlying neuromuscular disorders. 